\title{
How Localized Grounding, Combined with Conductive Skincare, Improves the Outcomes of the Traditional Skincare?
}

\author{
Jeremy Madvin and Madiha Khalid
}

\section{ABSTRACT}

\begin{abstract}
The main goals of acute and long-term skincare are the protection and improvement of skin health and integrity. Skincare involves several procedures that help maintain the skin's integrity, improve its appearance, and alleviate skin problems. Proper blood flow is in dire need for the skin to absorb vital ingredients. There are many conventional treatments to help increase blood flow and recently it was discovered that connecting the with the electrical charges of the Earth, or "Grounding" can promote healthy blood flow in the facial region. Additionally, such effects of grounding do not seem limited to the facial region but are rather systemic, essential and are often quickly observable. Grounding also seems to reduce secondary injury, collateral sprouting, and nerve sensitization. In grounded patients, an increase in blood flow was clearly seen when compared directly to ungrounded subjects. The results of documented studies explain that even short contact with the Earth helps to restore blood flow regulation suggesting enhanced skin tissue repair and improved skin appearance with possible implications for overall health.
\end{abstract}

Keywords: Earthing, Grounding, Blood Flow, Skincare.
Submitted : June 14, 2021

Published : July 10, 2021

ISSN: $2593-8339$

DOI: $10.24018 /$ ejmed.2021.3.4.935

\section{Jeremy Madvin}

MBA International Business, The University of Redlands, United States. (e-mail: jeremymadvin@gmail.com) Dr. Madiha Khalid *

Humdard University, Islamabad Campus, Pakistan.

(e-mail: msmadihakhalid@ gmail.com)

*Corresponding Author

\section{INTRODUCTION}

The largest living organ, the skin, defends the body from the outside world by regulating temperature and fluid balance, keeping pathogenic organisms and chemicals out, and providing sun protection. The stratum corneum, the outmost layer of skin, is a selectively permeable, heterogeneous layer of the epidermis that protects against desiccation and environmental challenges while retaining skin integrity. Damage to this layer results in loss of skin integrity, and chances of infection increase [1].

The main goals of acute and long-term skincare are protecting and improving skin health and integrity. Skin integrity is a quality indicator [2], and it is generally agreed that preserving skin integrity is more cost-effective than wound treatment [3]. Patients who are seriously or chronically ill and those who are immobile or have incontinence are at risk for various skin problems, including incontinence-associated dermatitis, pressure ulcers, intertriginous dermatitis, and skin drops. Age advancement can be considered an independent risk factor for developing skin problems due to ongoing changes in skin and associated soft tissue function and structure [4]. Skincare routines are often based on personal preferences, beliefs, and local care strategies instead of current best evidence-based practices [5].

Skincare is mainly a treatment of wound healing with utilizing radiation, medications, or some other method designed for prevention of future skin problems. Skin Care is a traditional medical discipline between cosmetics and dermatology [6]. The Federal Act on Food, Drugs and Cosmetics defines cosmetics as products of beauty and cleanliness (for instance, shampoos and lipstick) [7]. And dermatology involves medicines designed to diagnose, cure, mitigate, treat, or prevent disease or affect body structure and function (for example, sunscreens and acne creams); however, several other products are also present in this category, such as sunscreens, moisturizers and anti-dandruff formulations.

Traditionally, skincare differs from applied dermatology because it has a broader but lesser medical component and comprises non-medical professionals such as aestheticians, CAM specialists, and nurses [8]. Skincare includes changes in individual behavior, environmental conditions, and working conditions.

Precise definitions of skin care vary geographically. In America, skincare is a separate discipline, whereas it has been co-opted by dermatology in the U.K.

Skincare technology is diverse, and, because of the functionality of many skincare products, it differs from other cosmetic groups. There are many beauty products and pharmacological products with the common denominator being that these items are made to interact with and support the body's largest organ — the skin.

Skincare, however, involves several procedures that help maintain the skin's integrity, improve its appearance, and 
alleviate skin problems. They may include seemingly indirect approaches such as a balanced diet, avoiding unnecessary sun exposure, and using emollients appropriately. Cosmetics, exfoliation, botulinum toxin, laser resurfacing, fillers, microdermabrasion, retinol therapy, peels, and ultrasonic skin treatment are methods for improving skin appearance. Skincare may also exist as a routine of daily practices depending on the situation, including skin too dry or too moist, dermatitis prevention, and skin injury prevention.

Increased blood flow in your body helps to revitalize the skin and can even help delay the need for cosmetic surgery, (facelift), Botox, concentrated platelets, and Restylane injections. There are many treatments available to increase blood flow. Some people use cream with specific ingredients, while other use direct skin stimulation (ultrasound, massages, and lasers), chemical peels, and dermabrasion [9]-[10]. While these therapies produce results, they can have significant short- or long-term side effects. Many techniques that encourage the remodeling and synthesis of the dermal matrix and collagen synthesis require extensive post-treatment care and may cause complications, such as pain, infections, bleeding, erythema, oozing, burning, and scaring [10]. On the other hand, localized facial earthing, instead of being a complex procedure, requires little or no effort and can easily be integrated into everyday life.

The use of grounding for medicinal purposes is as old as humanity, and, in the coming years, we will likely see the continuation of the emergence on the market of new products using earthing principle. Earthing remains particularly well suited for further research. However, the use of earthing requires paying particular attention to the combination with other procedures. Additionally, the use of earthing in skincare products is highlighted by consumer demand, who are increasingly concerned with buying ecologically friendly products [10].

\section{A. Earthing (Grounding)}

The term "grounding" refers to the human body's link to the Earth's regular and active electric charges. It is a healthy lifestyle activity that impacts the body's basic bioelectrical functions on a systemic level. Grounding has been demonstrated to significantly improve physiology, reduce pain and inflammation, boost blood flow and venous/ lymphatic flow back to the heart, improve sleep, and reduce stress and anxiety, all of which contribute to a sense of wellbeing. There also appear to be significant structural effects. Based on countless reports, after grounding, many people say they feel and look healthier and happier. Many people who are in pain find that grounding helps them feel better [11]. Morale is even increased [12]. Grounding is a simple process that reportedly and frequently yields quick results, especially for persons with chronic health issues.

Grounding can be accomplished in two ways:

1) Indoor use grounding devices,

2) Outdoors, barefoot directly upon the earth's surface.

Grounding restores a primaeval electrical connection with the planet. Because modern humans have an evolved lifestyle based in artificial surroundings, flooring and shoes, grounding appears to help with "electron deficiency syndrome," a neglected and possibly primary cause of a variety of health problems [13]. Grounding appears to be a strong tool for illness prevention and rehabilitation. This technique can be used alone or in combination with conventional therapies to help relieve symptoms and eradicate the fundamental causes of diseases and conditions that begin with a bioelectric imbalance in the body. The medical community needs to understand further that grounded body is probably healthier than that of an ungrounded body. Simply said, the body performs more effectively and with less cellular stress. Humans have become increasingly estranged from the planet. Obviously, we do not usually sleep in the grass, we often do not go out without shoes and have worn almost exclusively plastic isolating sole shoes instead of traditional and conductive leather shoes for more than half a century. We live and work in buildings that are elevated and sometimes hundreds of feet above ground and spend most of our time electrically separated from the earth.

A lack of contact with the Earth can lead to electrical imbalances, the formation of hazardous positively charged ions, and an unknown body depletion of electrons, as well as vulnerability to malfunction, infection, and disorder. Solar radiation, uncountable electrical interactions such as thunderbolts, and other dynamic forces of the environment all have an impact on Planet Earth's surface, as is generally understood. However, it is not widely known that these events provide a constant supply of free electrons to land masses and aquatic bodies, allowing the Earth to maintain a continuous negative electrical charge. Biological earth science says that this same electric charge serves a regulative and supporting purpose both of animals and plants kingdom, similar to "electrical nutrition," with its continuous electron availability and circadian frequencies. These findings support the hypothesis that grounding permits a large number of free electrons to enter the body, causing rapid, frequently instantaneous physical changes. Earthing preserves and maintains a normal internal electrical situation. Sokal and Sokal, two Polish researchers and medics, conducted several studies and came to the conclusion that the human body is a "universal regulating force in Nature". Biochemical, Bioelectric, and bioenergetic processes are all strictly monitored, and they have a big influence on chronic illnesses [14].

\section{MODERN CONCEPTS OF EARTHING IN SKINCARE}

External physical stimulus can activate electrochemical reactions that affect vital biological processes in the body. In oriental medicine, grounding therapy is also viewed as the initiation of such a reaction, causing unbalanced physiological states to return to its normal physiological equilibrium. The theory of physiological therapy in Modern medicine has been explained as a morphological and hormonal control process [16]. Earthing may lead to brain reactions; not all responses may result in brain responses because the natural response to environmental stimuli is considerably faster than is conceivable through the neurological system. It suggests that biological mechanisms other than the nervous system may be at action [17].

Stimulation of the skin while earthing usually produces an ion-electric-charge, which is transferred via numerous biological pathways — blocking the ion-electric-charge 
causes an unequal negative and positive charge in a body, resulting in pain and the onset of chronic disease. Earthing helps to restore good health by moving the excessive or inadequate condition of the ion-electric charge to or from the body based on the body's natural pursuit of electrical balance with the earth. It indicates that the transmission of bio-electric charges is closely connected with earthing. It immediately follows that a combo therapy of skincare with simultaneous grounding offers an increase in probability of positive benefits without complications.

The extrinsic or intrinsic factors takes results in free radicals' formation in the skin that damages the skin. Free radicals are unstable molecules that take electrons from the skin, when electrons are taken away from the skin it damages the repair process of the skin. Anti-oxidants in the skin helps prevent damage and maintains healthy skin. Skincare products possess anti-ageing, anti-inflammatory and antiirritative properties. Anti-oxidants acts as a shield for the skin provide free electrons to the skin to repair the damage. Earthing is a natural anti-oxidant that transfer electrons to the body and maintains the skin texture.

Anti-inflammatory ingredients in skincare products reduce pain, swelling and redness in the skin that prevents acne. Increased blood flow reduces the pain and tighten the muscles and allows the inflammatory response to happen easily to cure the inflammation.

Because of its negligible negative effects and recorded therapeutic success, grounding therapy has become a popular primary therapeutic technique. Despite the expanding number of studies, the role of earthing in skincare has still not been thoroughly investigated but individual case studies are promising. Both techniques are used in conjunction to obtain the synergistic effect. This technique produces cautious optimism, one which localized blood flow, as well as efficiency and effectiveness of topical ingredients improve dramatically.
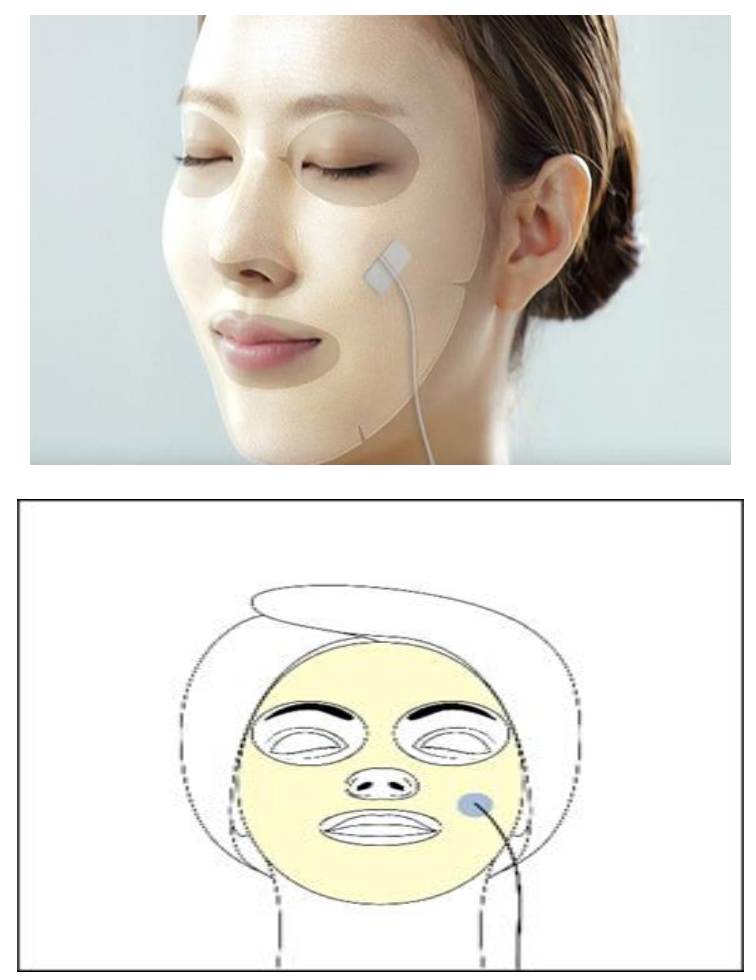

Fig.1. Skincare earthing device.
The above picture is of specially engineered grounding skin patches that acts as a grounding device that injects electrons into body using only a grounding port. However, if outside power sources are used and a stronger current is applied, this can result in harm to the tissue. For effective facial grounding, first, a conductive skincare topical is applied for moisturizing, cleansing, hydrating, etc. The topical defines where the body's voltage exits, and the Earth's ions enter. Then a grounding connection is applied to the face or other body part being treated with skincare. The patient can choose precisely where this exchange occurs (entire face, neck, under the eyes, and so on). Grounding has been demonstrated to increase microcirculation in the face. These devices provide patients a lot of flexibility in terms of dealing with individual symptoms while also addressing more general inflammatory or immunological disorders. To maximize stimulation and aid in the unblocking procedure, the user could choose the path and exit point for the cell's excess voltage and send it through a specific portion of the body or muscle group.

This process of using earthing in skincare likely increases blood flow to the specific region of the organ being grounded. Another well-documented benefit of earthing in skincare is improved blood flow. The zeta potential on the surface of red blood cells is a negatively charged intensity that preserves the distance between blood cells. The higher the charge on the blood is more, it increases the viscosity of blood and the better the flow, the increased the ability of the cells to push one another (less clumping). This indicates an average bloodthinning effect. The researchers found that earthing reduces blood clogging and viscosity, so it appears to be among the most approachable, in-depth methods to help lower the risk of developing cardiovascular disease [18].

Users will also benefit from the fact that these operations may be completed faster, allowing them to get in and out of the clinic faster. According to studies, the advantages of localized grounding are best after only 25 minutes.

Using a Speckle Contrast Laser camera, Chevalier obtained image proof in 2014 that earthing increases blood circulation to the face and improves control of the autonomic nervous system on peripheral circulation. When earthing subjects were compared to non-earthing subjects, the researchers identified an intriguing and different pattern in their facial blood flow [19]. This pattern connects to the ANS's improved blood flow management and explains how earthing improves facial skin and tissue regeneration and recovery, resulting in a beauty effect.

The cosmetics industry uses a variety of therapies to increase facial microcirculation. Fig. 2 shows imaging results of a study.

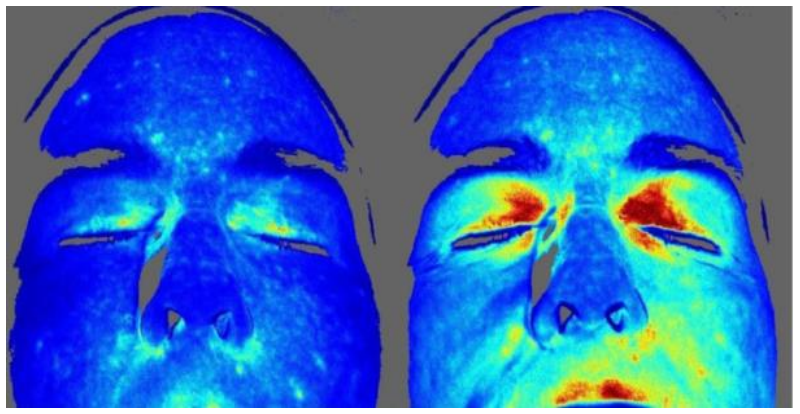

Fig. 2. Blood circulation to face area improved with earthing. 
According to Chevalier, earthing improves mechanism like increased blood fluidity, regulated levels of cortisol, which calm the body, lower swelling, and improved general body physiology.

Additional and substantial benefits of earthing include stress reduction and mood enhancement, which are achieved through a stabilizing influence on nervous system and the stress hormone [20].

A combination of the impacts outlined in this article are likely to influence blood pressure. Several case studies have been published that reveal a significant reduction in blood pressure once they start on regular grounding sessions [21]. Patients participated in the first study in which they evaluated this link experienced a consistent improvement of blood pressure within a couple of months and were well-balanced in several cases within a limited timeframe.

Thermal imaging was used in a 2015 study to record the increased blood flow in the torso caused by earthing [22]. From the torso, blood travels to the head and face through the throat. As More blood travels to the face, there is likely an increase in the brain's blood flow. The torso lymphatic fluid circulation was increased, according to the imaging analysis.

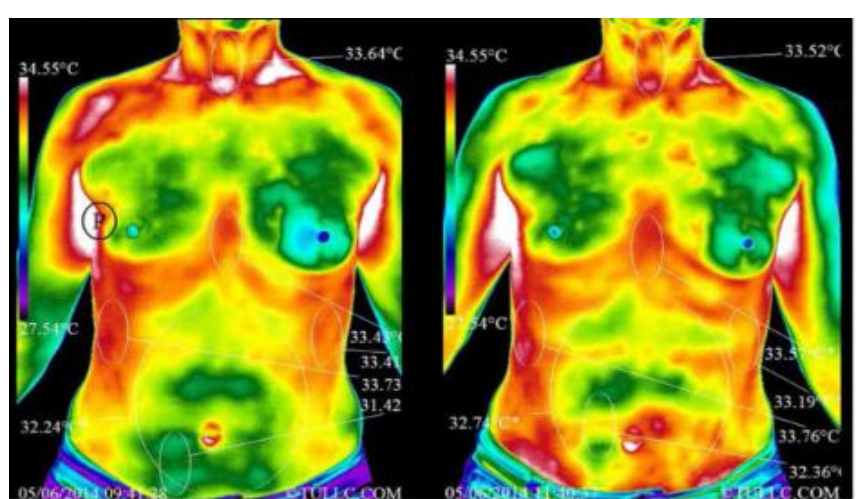

Fig. 3. Temperature difference was seen in the figure of earthing of $55 \mathrm{yr}$ older woman. Before earthing (left) and one hour after earthing (right).

Photographic images of a non-healing wound's recovery (Fig. 4) provide yet another remarkable example of fast recovery from grounding. It demonstrates the rapid healing of an 8th-month-old non-healing wound caused by a few hours of wearing a tight-fitting boot. During that time, a sore developed, which progressed to a resilient, open wound. Several procedures at a specialist wound center proved ineffective. The patients' legs had poor circulation, according to vascular imaging. The patient had a slight limp and was in agony when she first saw a doctor who gave grounding treatments. The patient was grounded for two weeks in the clinic for 30 minutes per day with an earthing patch while sitting comfortably. She experienced a substantial reduction in pain after the first session. She noticed the pain had decreased by around $80 \%$ after one week. She said she was pain-free after two weeks.
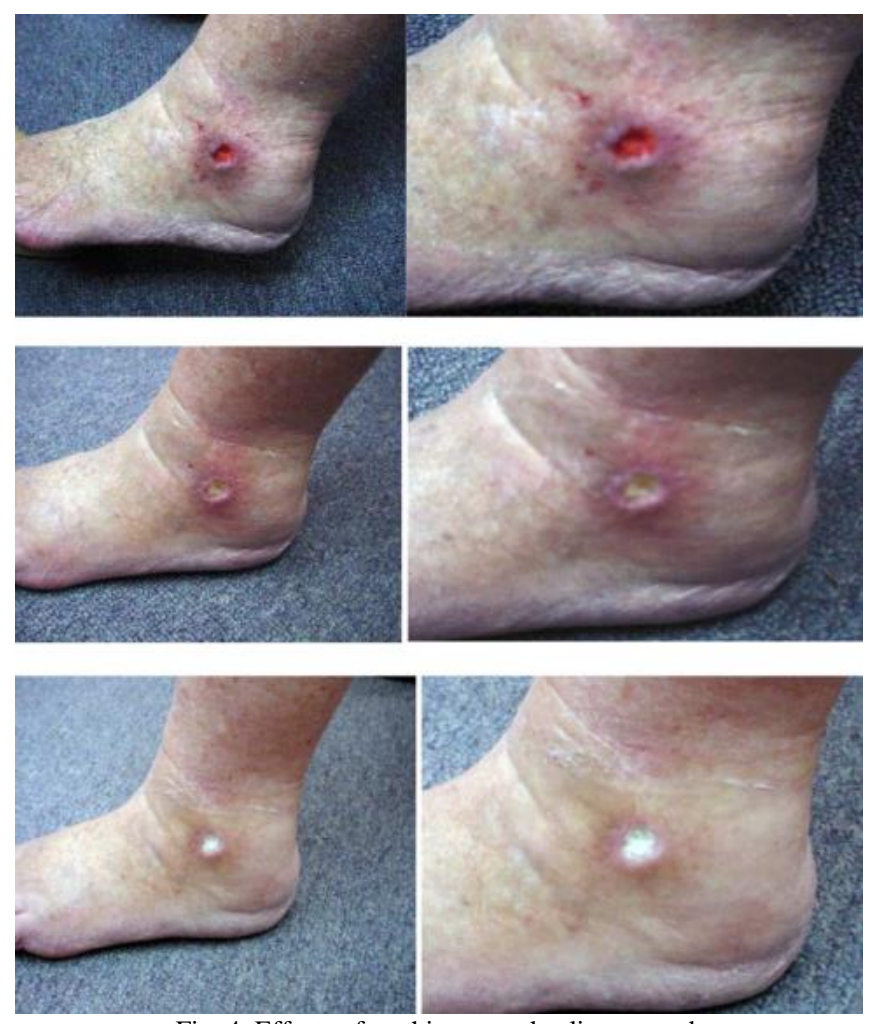

Fig. 4. Effects of earthing on unhealing wound.

\section{DISCUSSION}

This study was aimed at determining whether the earthing of the body promotes B.F. The aim of various treatments used in the beauty industry is to improve microcirculation [23]. Improved skin circulation can lead to increased skin nutrition through increased oxygen and nutrient delivery and increased protection against the oxidative aging process [24]. Therefore, the current results suggest a more effective ANS regulation of the B.F. is supported in connection with the grounding. Earthing restores blood circulation by the ANS and, as a result, different organs receive oxygen and nutrients accordingly to their needs. Another reason for improving BF is the Zeta Potential (Z.P.) and red blood cell aggregation (RBCs). Several clinical situations, including hypertension, lipid disorders, diabetes Mellitus, smoking, and increasing age, are associated with increased viscosity in the blood. The lower the RBC surface, the higher the repellence between RBCs, which means lower blood viscosity leading to increased blood flow [25]. Grounding Markedly enhances Z.P. and decreases RBC aggregation.

A third explanation for improved B.F. shows how grounding helps to improve physiology. This statement supports that earthing may improve wound recovery, calcium metabolism, thyroid function, cell glucose use, immunoresponse, and oxygen consumption [26]. It also appears helpful in reducing physical feelings of stress. Many studies show that sleep disorders caused by significant stress impact skin functions and integrity [27]. Previous grounding studies have resulted in better sleep subjectively reported by grounded participants and less stress [28]. Grounding also seems to enhance the balance of the sympatheticparasympathetic mechanism of ANS; this also further affects stress reduction [29]. In addition, grounding has been documented for inflammation reduction through various measurements [30]. Skin aging is the main effect of oxidative 
stress [31]. Oxidative stress breaks down protein, alters cell renewal cycles, increases cross-linking of proteins, causes DNA damage, and releases pro-inflammatory mediators, which cause inflammatory diseases of the skin. Free radicals also cause allergic reactions. Grounding action may also aid in reducing skin damage from U.V. radiation which produces oxidative stress [32]. The result of this research indicates that periods of extended grounding could result in further changes and benefits. Grounding must be included in complementary, preventive, and lifestyle clinical training as many of these techniques can be needs to be reached.

\section{CONCLUSION}

Localized grounding, when incorporated into a conventional skincare regimen is a non-pharmacological and a non-invasive intervention with a wide range of functions and benefits. The Earth in which we live has a form of natural electrical energy that is easy to access and beneficial to human physiology in many ways [6]. New research backs up previous findings, indicating that grounding may be effective in the treatment of a variety of acute and chronic disorders. Proper blood flow is in dire need by the skin to absorb vital ingredients. Grounding can deliver generous benefits of not only increased blood flow; however, pain reduction and other favorable results are achieved without causing substantial side effects. This study backs up these findings by demonstrating a considerable increase in self-regulation of facial blood flow in grounded patients but not in shamgrounded people. The findings reveal, first, that even a brief touch with Earth restores blood flow regulation to the face, which can aid in skin tissue repair, improved health, and vitality, and improved facial beauty.

While incorporating grounding and skincare into a single system shows great promise, more research is needed, with additional measuring techniques, to validate the Earth's novel role as a skin health and appearance protector.

\section{REFERENCES}

[1] Rawlings AV, Scott IR, Harding CR, Bowser PA. Stratum corneum moisturization at the molecular level. J Invest Dermatol. 1994 Nov;103(5):731-41. doi: 10.1111/1523-1747.ep12398620. PMID: 7963664.

[2] Nakrem, S., Vinsnes, A. G., Harkless, G. E., Paulsen, B., \& Seim, A. (2009). Nursing sensitive quality indicators for nursing home care: international review of literature, policy and practice. International Journal of Nursing Studies, 46(6), 848-857.

[3] Beeckman, D., Schoonhoven, L., Verhaeghe, S., Heyneman, A., \& Defloor, T. (2009). Prevention and treatment of incontinenceassociated dermatitis: literature review. Journal of advanced nursing, 65(6), 1141-1154.

[4] Kottner, J., Lichterfeld, A., \& Blume-Peytavi, U. (2013). Maintaining skin integrity in the aged: a systematic review. British Journal of Dermatology, 169(3), 528-542.

[5] Farage, M. A., Miller, K. W., Elsner, P., \& Maibach, H. I. (2008). Functional and physiological characteristics of the aging skin. Aging clinical and experimental research, 20(3), 195-200.

[6] Kessler R. More than Cosmetic Changes: Taking Stock of Personal Care Product Safety. Environ Health Perspect; DOI:10.1289/ehp.123 A12.

[7] FDA. Cosmetics: Guidance \& Regulation; Laws \& Regulations. Prohibited \& Restricted Ingredients. [website]. U.S. Food and Drug Administration, Silver Spring, MD. Updated 26 January 2015.

[8] Schwanitz, H., Riehl, U., Schlesinger, T., Bock, M., Skudlik, C., \& Wulfhorst, B. (2003). Skin care management: educational aspects.
International Archives of Occupational and Environmental Health, 76, 374-381.

[9] Med-Health.net (2014) How to Tighten Face Skin. http://www.medhealth.net/How-To-Tighten-Face-Skin.html.

[10] Herman, J., Rost-Roszkowska, M. and Skotnicka-Graca, U. (2013) Skin Care during the Menopause Period: Noninvasive Procedures of Beauty Studies. Postẹpy Dermatologii i Alergologi, 30, 388-395. http://www.ncbi.nlm.nih.gov/pmc/articles/PMC3907896/.

[11] Avci, P., Gupta, A., Sadasivam, M., Vecchio, D., Pam, Z., Pam, N. and Hamblin, M.R. (2013) Low-Level Laser (Light) Therapy (LLLT) in Skin: Stimulating, Healing, Restoring. Seminars in Cutaneous $\begin{array}{lll}\text { Medicine and } & \text { 32, }\end{array}$ http://www.ncbi.nlm.nih.gov/pmc/articles/PMC4126803/.

[12] Ober C, Sinatra ST, Zucker M. Earthing: the most important health discovery ever? Basic Health Publications, Inc.; 2010.

[13] Chevalier G. The effect of grounding the human body on mood. Psychological Reports. 2015 Apr;116(2):534-42.

[14] Oschman, J. L., Chevalier, G., \& Brown, R. (2015). The effects of grounding (earthing) on inflammation, the immune response, wound healing, and prevention and treatment of chronic inflammatory and autoimmune diseases-Journal of Inflammation Research, 8, 83.

[15] Sokal, K., \& Sokal, P. (2011). Earthing the human body influences physiologic processes. The Journal of Alternative and Complementary Medicine, 17(4), 301-308.

[16] Guyton AC. Nervous regulation of the circulation and rapid control of arterial pressure. Textbook of medical physiology. 1991:194-204.

[17] Becker, Robert O. "Exploring new horizons in electromedicine." (2004): 17-18.

[18] Chevalier G, Sinatra ST, Oschman JL, Delany RM. Earthing (grounding) the human body reduces blood viscosity - a major factor in cardiovascular disease. The journal of alternative and complementary medicine. 2013 Feb 1;19(2):102-10.

[19] Chevalier, G. (2014). Grounding the human body improves facial blood flow regulation: results of a randomized, placebo-controlled pilot study - Journal of Cosmetics, Dermatological Sciences and Applications, 4(05), 293.

[20] Ghaly, M., \& Teplitz, D. (2004). The biologic effects of grounding the human body during sleep as measured by cortisol levels and subjective reporting of sleep, pain, and stress. Journal of Alternative \& Complementary Medicine, 10(5), 767-776.

[21] Elkin, H. K., \& Winter, A. (2018). Grounding Patients with Hypertension Improves Blood Pressure: A Case History Series Study. Altern Ther Health Med, 24(6), 46-50.

[22] Chevalier, G., Melvin, G., \& Barsotti, T. (2015). One-hour contact with the Earth's surface (grounding) improves inflammation and blood flow-A randomized, double-blind, pilot study. Health, 7(08), 1022.

[23] Avci, P., Gupta, A., Sadasivam, M., Vecchio, D., Pam, Z., Pam, N. and Hamblin, M.R. (2013) Low-Level Laser (Light) Therapy (LLLT) in Skin: Stimulating, Healing, Restoring. Seminars in Cutaneous Medicine and Surgery, 32, 41-52.

[24] Schwartz, S.R. and Park, J. (2012) Ingestion of BioCell Collagen ${ }^{\circledR}$, a Novel Hydrolyzed Chicken Sternal Cartilage Extract; Enhanced Blood Microcirculation and Reduced Facial Aging Signs. Clinical Interventions in Aging, 7, 267-273.

[25] Chevalier, G., Sinatra, S.T., Oschman, J.L. and Delany, R.M. (2013) Earthing (Grounding) the Human Body Reduces Blood Viscosity: A Major Factor in Cardiovascular Disease. Journal of Alternative and Complementary Medicine, 19, 102-110.

[26] Ober, C., Sinatra, S.T. and Zucker, M. (2010) Earthing: The Most Important Health Discovery Ever? Basic Health Publications, Laguna Beach, 193-205.

[27] Gupta, M.A. and Gupta, A.K. (1996) Psychodermatology: An Update Journal of the American Academy of Dermatology, 34, 1030-1046.

[28] Rööst, M. and Nilsson, P. (2002) Sleep Disorders-A Public Health Problem. Potential Risk Factor in the Development of Type 2 Diabetes, Hypertension, Dyslipidemia and Premature Aging. Läkartidningen, 99, 154-157.

[29] Chevalier, G. and Sinatra, S.T. (2011) Emotional Stress, Heart Rate Variability, Grounding and Improved Autonomic Tone: Clinical Applications. Integrative Medicine: A Clinician's Journal, 10, 16-21.

[30] Oschman, J.L., Chevalier, G. and Brown, D. (2014) The Effects of Grounding (Earthing) on Inflammation, the Immune Response, Wound Healing and Prevention and Treatment of Chronic Inflammatory and Auto-Immune Diseases. Journal of Inflammation Research, in Press.

[31] Masaki, H. (2010) Role of Antioxidants in the Skin: Anti-Aging Effects. Journal of Dermatological Science, 58, 85-90.

[32] Burke, K.E. and Wei, H. (2009) Synergistic Damage by UVA Radiation and Pollutants. Toxicology and Industrial Health, 25, 219224. 
European Journal of Medical and Health Sciences www.ejmed.org

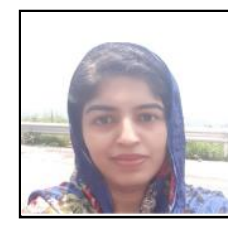

Madiha Khalid

Place of birth: Lahore.

Date of Birth: 11/9/1985.

M. Phil clinical Pharmacy, Humdard University,

Lahore.

LECTURER in THE UNIVERSITY OF

LAHORE for about 6 years in the Pharmacy

Department.

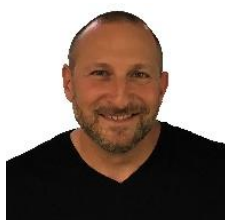

\section{Jeremy Madvin}

Place of Birth: Los Angeles.

Bate of Birth: 11/22/1967.

MBA International Business, University of Redlands,

United States. 